\title{
Heart Failure in Which Coronary Spasms Played an Important Role
}

\author{
Shinsuke Oda ${ }^{1}$, Yuichi Fujii ${ }^{1}$, Hiroaki Takemoto ${ }^{1}$, Shuichi Nomura ${ }^{1}$, Hirofumi Nakayama ${ }^{2}$, \\ Yasushi Toyota $^{3}$, Hiroshi Nakamura ${ }^{4}$ and Hiroki Teragawa ${ }^{1}$
}

\begin{abstract}
A 69-year-old woman was admitted for further examinations and treatment of chest pain. Emergency cardiac catheterization showed no significant stenosis on coronary angiograms; however, diffuse wall hypokinesis was observed on a left ventriculogram. After treating the patient's heart failure, cardiac catheterization was performed again. A spasm provocation test showed coronary spasms of the right and left coronary arteries. A right ventricular endomyocardial biopsy revealed denaturation and fibrosis of the myocardium under the endocardium, thus suggesting the presence of myocardial ischemia. This case highlights coronary spasms as a cause of heart failure.
\end{abstract}

Key words: vasospastic heart failure, coronary spasm, acetylcholine

(Intern Med 53: 227-232, 2014)

(DOI: 10.2169/internalmedicine.53.1217)

\section{Introduction}

Vasospastic angina is characterized by transient abnormal contractions of the epicardial coronary arteries, causing myocardial ischemia and anginal attacks (1-3). It is well known that coronary spasms cause not only anginal attacks, but also fatal arrhythmia and sudden death (4). Contrary reports have also stated that coronary spasms may play a role in the development of heart failure (5-8). In this study, we report the case of a patient with heart failure due to coronary spasms, as suggested by the clinical and pathological findings.

\section{Case Report}

A 69-year-old woman was being regularly followed up by her outpatient physician during the course of treatment for chronic atrial fibrillation and hypothyroidism. Echocardiography performed five months before admission to our hospital revealed a left ventricular ejection fraction (LVEF) of $50 \%$. In November 2011, she experienced general mal- aise due to excitement. In early December 2011, she began to experience a feeling of chest oppression while using the stairs. Toward the end of December, she experienced chest pain that lasted for 30 minutes after bathing at approximately 21:00 hours. At midnight, she experienced chest pain during sleep for 30 minutes. When she consulted her family physician the next day, an electrocardiogram (ECG) showed ST changes, and a human-fatty acid binding protein assay was positive. Therefore, she was referred to our hospital and admitted for a further examination.

The patient had a family history of hypertension, gastric carcinoma and glaucoma on her father's side. She also had a history of ectopic pregnancy and acute pyelonephritis at 25 years of age and glaucoma at 67 years of age. Her physical examination findings on admission were as follows: consciousness, clear; body temperature, $36.2^{\circ} \mathrm{C}$; blood pressure, $114 / 70 \mathrm{mmHg}$; pulse rate, 92 beats/min and irregular; resting $\mathrm{SpO}_{2}, 99 \%$ (indoor atmospheric pressure); rales in the lower lung fields; and no cardiac murmurs. Laboratory tests performed on admission revealed elevated levels of creatinine kinase (314 IU/L) and brain natriuretic peptide (299 $\mathrm{pg} / \mathrm{mL}$ ) with a positive quick troponin $\mathrm{T}$ test. On a chest $\mathrm{X}$ -

\footnotetext{
${ }^{1}$ Department of Cardiovascular Medicine, Hiroshima General Hospital of West Japan Railway Company, Japan, ${ }^{2}$ Department of Pathology, Hiroshima General Hospital of West Japan Railway Company, Japan, ${ }^{3}$ Mihara Clinic, Japan and ${ }^{4}$ Deparment of Community Health and Medicine, Yamaguchi University School of Medicine, Japan

Received for publication June 21, 2013; Accepted for publication September 9, 2013

Correspondence to Dr. Hiroki Teragawa, hiroteraga71@gmail.com
} 

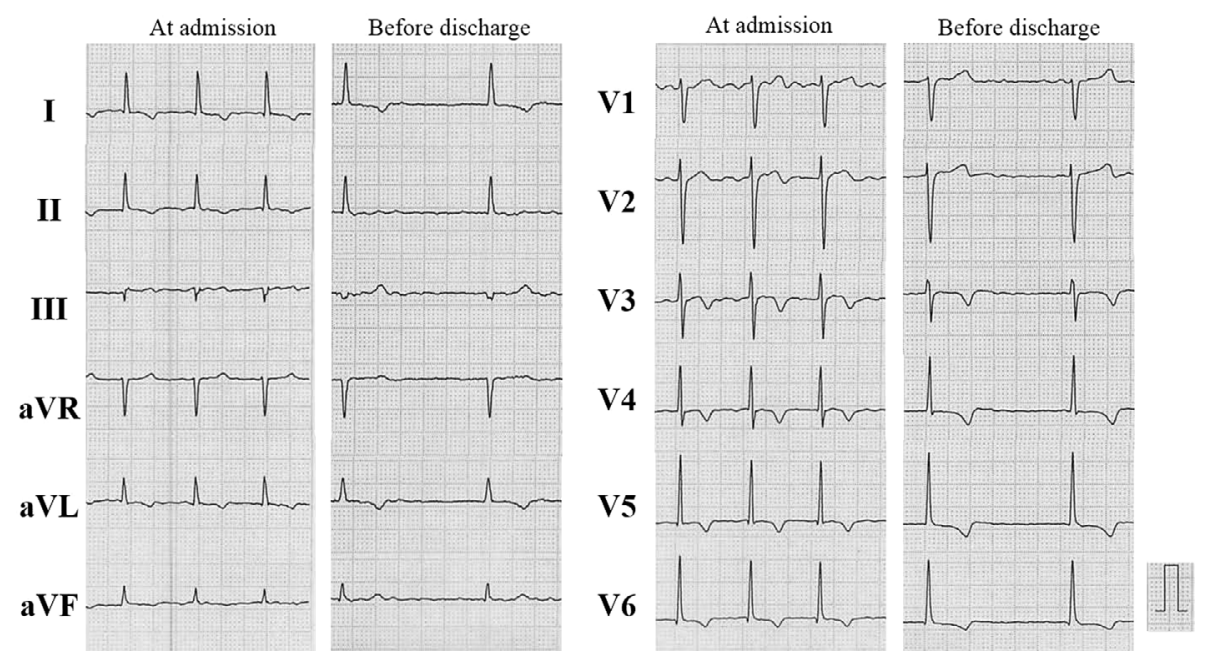

Figure 1. Electrocardiograms (ECGs) obtained on admission and before discharge. An ECG obtained on admission revealed atrial fibrillation and negative T waves in I, II, III, aVL, aVF and V3-6. An ECG obtained before discharge revealed bradycardia with atrial fibrillation and negative $T$ waves, as observed on the ECG obtained on admission.

(a) On admission

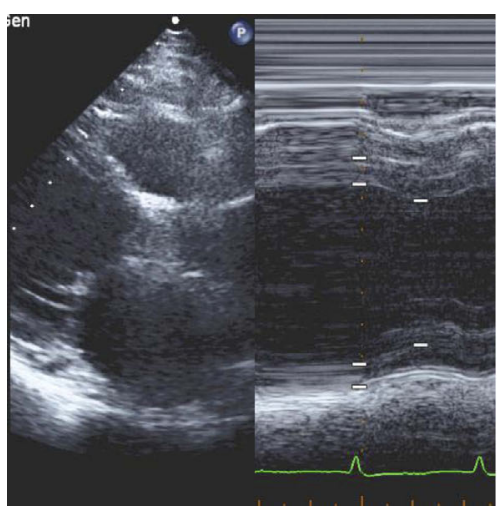

LVDd 57 mm, LVDs 46 mm, LVEF $39 \%$ (b) The 11th hospital day

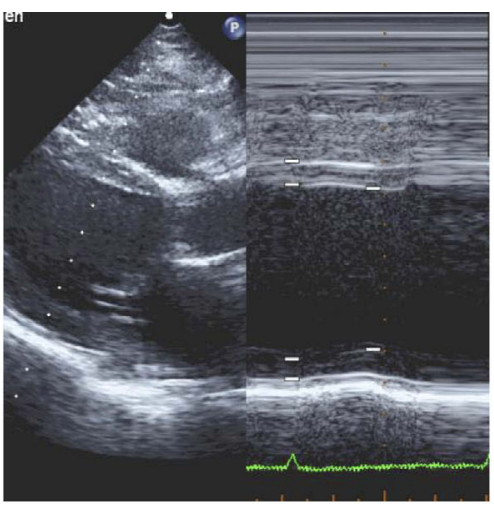

LVDd $55 \mathrm{~mm}$, LVDs $51 \mathrm{~mm}$, LVEF $16 \%$ (c) Before discharge

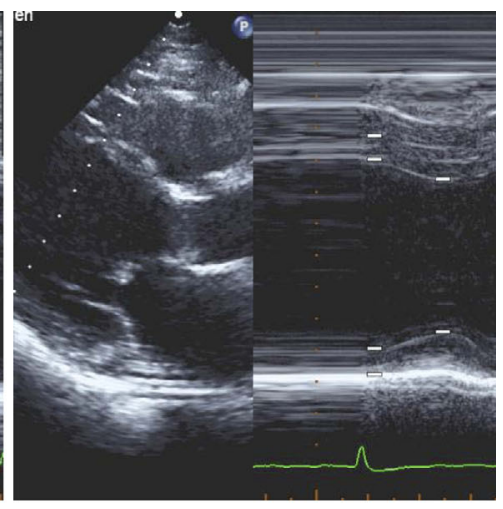

LVDd $60 \mathrm{~mm}$, LVDs $48 \mathrm{~mm}$, LVEF $40 \%$

Figure 2. Echocardiography performed on admission (a) revealed diffuse hypokinesis of the left ventricle (LV), with an LVEF of 39\%. Echocardiography performed on the 11th hospital day (b) revealed diffuse hypokinesis of the $L V$, with an $L V E F$ of $19 \%$, which was worse than that observed on admission. Echocardiography performed before discharge (c) revealed diffuse hypokinesis of the LV,

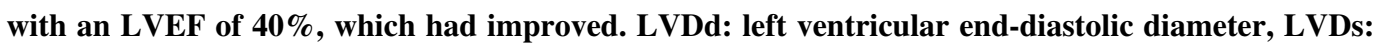
left ventricular end-systolic diameter, LVEF: left ventricular ejection fraction

ray, the cardiothoracic ratio was $63 \%$, indicating cardiomegaly, and slight congestion was observed in the bilateral lung fields. An ECG revealed atrial fibrillation and negative $\mathrm{T}$ waves in I, II, aVL, aVF and V3-6 (Fig. 1). Echocardiography revealed diffuse hypokinesis of the left ventricle (LV), with an LVEF of $39 \%$ accompanied by slight dilatation of the LV with an LV end-diastolic dimension of 57 mm (Fig. 2a). Emergency cardiac catheterization was performed. Coronary angiography indicated only moderate stenosis of the left anterior descending coronary artery (LAD), while left ventriculography (LVG) showed diffuse hypokinesis of the LV, with an LVEF of $26 \%$ (Fig. 3).
Heart failure was diagnosed based on the these clinical findings, although the causes of the patient's chest pain and elevation of the levels of cardiac biomarkers were unclear at the time. First, we administered a diuretic and beta blocker to treat the heart failure. On the fourth hospital day, adenosine stress thallium-201 myocardial perfusion scintigraphy revealed LV dilatation and diffuse impairment of the LV wall motion with no perfusion defects, suggesting myocardial ischemia or necrosis (Fig. 4). The status of the patient's heart failure improved, not promptly but rather gradually, in response to the treatment of heart failure. Echocardiography performed on the 11th hospital day revealed diffuse hypoki- 
(a)

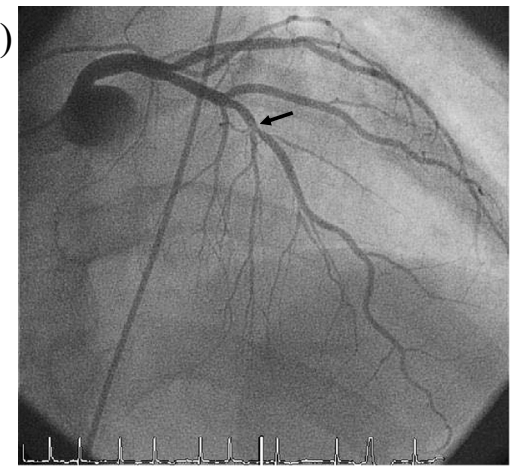

(b)

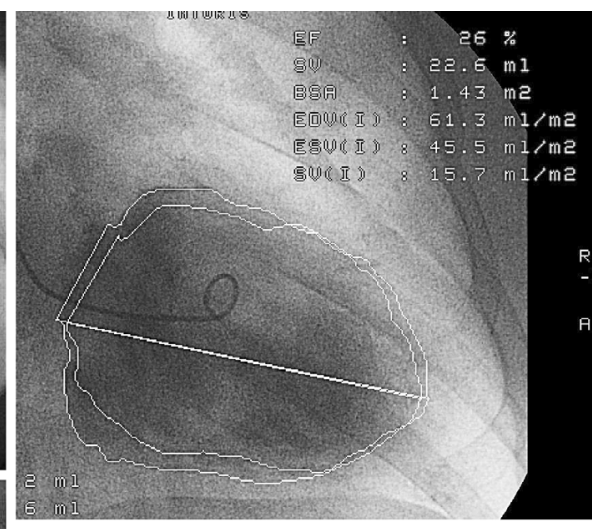

(c)

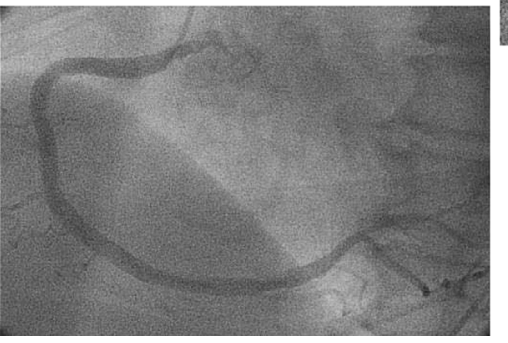

Figure 3. (a, b) Emergency coronary angiograms revealed moderate stenosis of the proximal segment of the left anterior descending coronary artery (LAD) without stenosis of the right coronary artery (RCA). (c) Left ventriculography revealed diffuse hypokinesis of the left ventricle (LV).

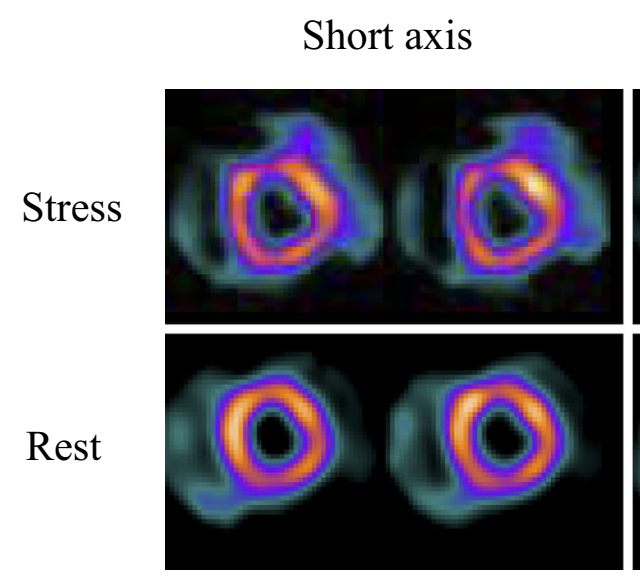

Vertical long axis
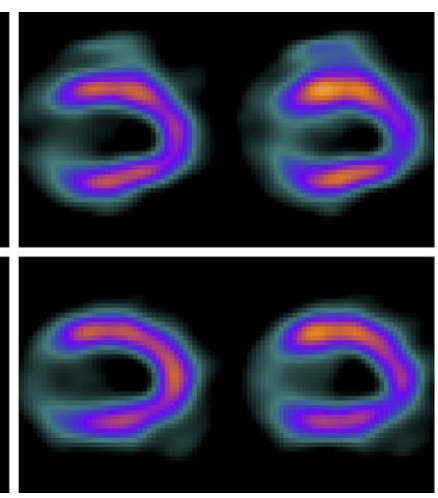

Horizontal long axis
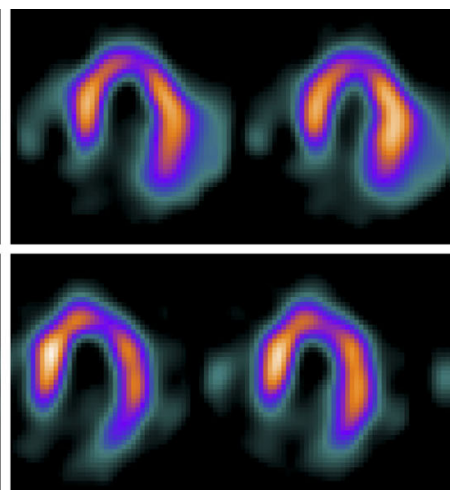

Figure 4. Adenosine stress thallium-201myocardial perfusion imaging showed dilatation of the left ventricle $(\mathrm{LV})$ without perfusion defects.

nesis of the LV with an LVEF of 19\%, which was worse than that observed at admission (Fig. 2b).

Because the causes of the patient's chest pain and increased cardiac enzyme levels were unclear, we performed cardiac catheterization again on the 17th hospital day. SwanGanz catheterization showed a normal pulmonary capillary wedge pressure $(10 / 8 / 9 \mathrm{mmHg})$ and a reduced cardiac index $\left(1.47 \mathrm{~L} / \mathrm{min} / \mathrm{m}^{2}\right)$. Coronary angiography showed the same findings as the first examination; therefore, a coronary spasm provocation test was performed. Infusion of acetylcholine at doses of 30 and $50 \mu \mathrm{g}$ caused severe spasms of the distal portion of the right coronary artery, accompanied by chest pain. Due to the prolonged coronary spasms, $5 \mathrm{mg}$ of nitroglycerin was administered via an intracoronary infusion. Thereafter, the coronary spasms were alleviated (Fig. 5). Subsequently, the infusion of acetylcholine at doses of 50 and $100 \mu \mathrm{g}$ caused diffuse spasms of the mid-distal segments of the LAD and focal spasms of the proximal segment of the left circumflex coronary artery, accompanied by chest pain. Following the administration of nitroglycerin, the coronary spasms were alleviated, and no significant stenosis was observed in the coronary arteries (Fig. 5). LVG showed diffuse impairment of the LV, with an LVEF of $41 \%$. To investigate the cause of the LV systolic dysfunction, we performed a right ventricular endomyocardial biopsy. The degree of endomyocardial fibrosis varied from one area to another. The myocardial fibers were in disarray and differed in size with nuclear irregularities (Fig. 6a). Mild myocardial fibrosis was also observed (Fig. 6b).

Based on these findings, we diagnosed the patient as having vasospastic angina with repeated myocardial ischemia caused a denatured myocardium, leading to the development 

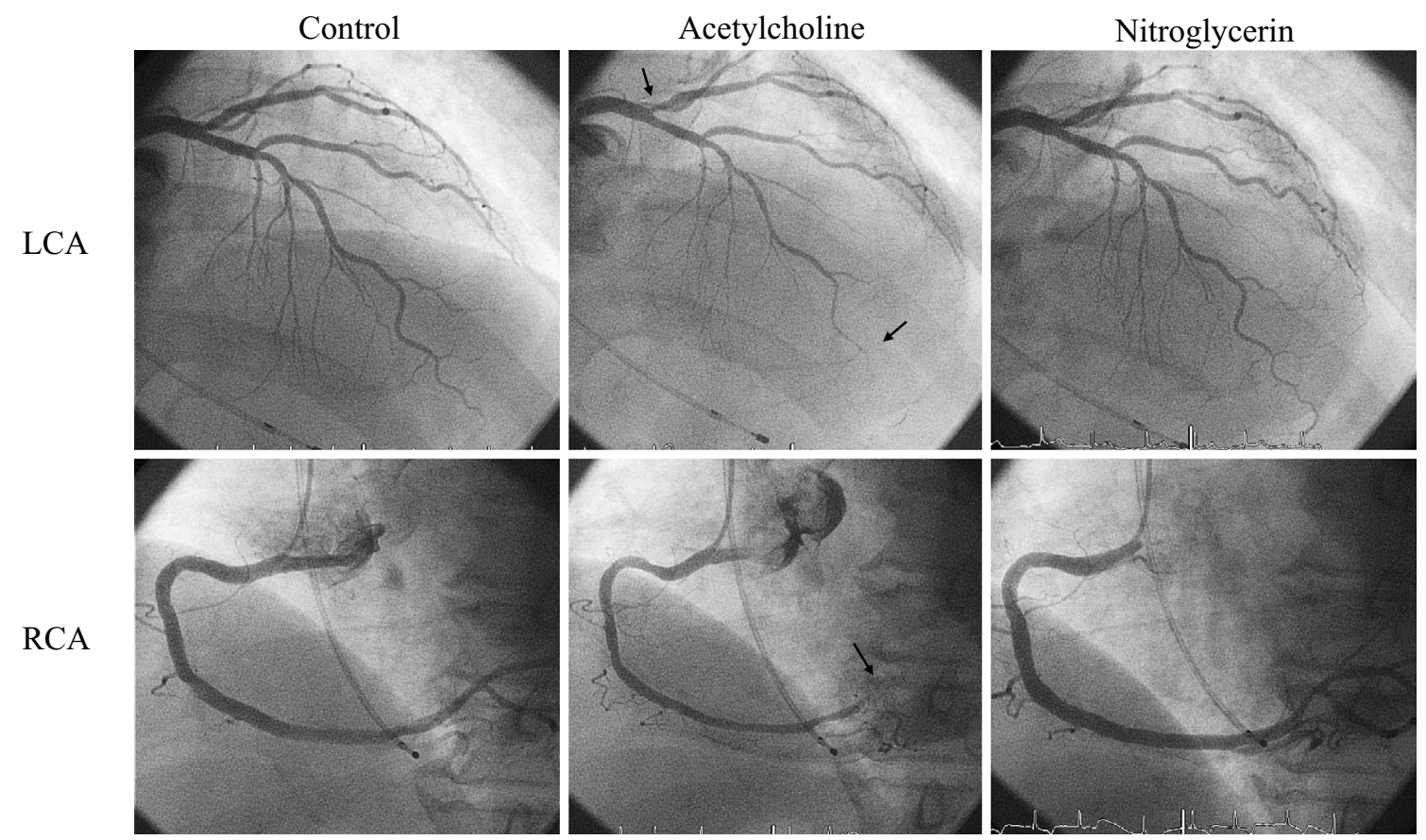

Figure 5. A second coronary angiogram showed no significant coronary stenosis on the control images (left), subtotal spasms of the distal segment of the left anterior descending coronary artery (LAD) and moderate spasms of the proximal segment of the left circumflex coronary artery during the infusion of acetylcholine (center; the spasm sites are indicated by arrows) in addition to the disappearance of the coronary spasms following the intracoronary infusion of nitroglycerin (right).

(a)

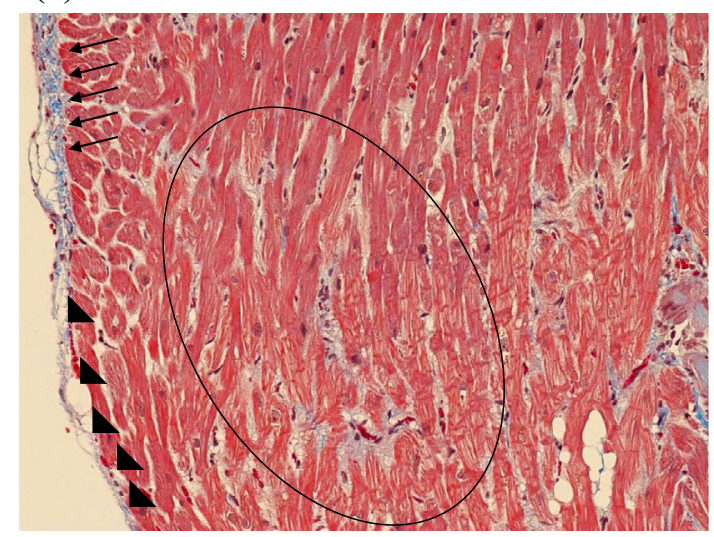

Masson-trichrome stain $\times 200$ (b)

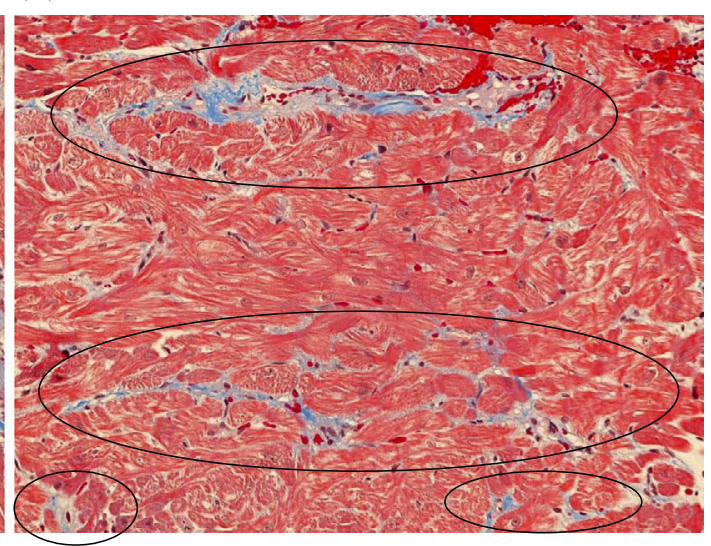

Masson-trichrome stain $\times 400$

Figure 6. An endomyocardial biopsy showed (a) endomyocardial fibrosis that varied from one area (arrows) to another (arrowheads). The myocardial fibers exhibited disarray (circle) and differed in size with nuclear irregularities. (b) Mild myocardial fibrosis was observed (circles).

of heart failure. Calcium channel blockers were administered. Before discharge on the 22nd hospital day, an ECG showed bradycardia with atrial fibrillation and negative $\mathrm{T}$ wave changes similar to that observed at admission (Fig. 1). Echocardiography revealed diffuse hypokinesis of the LV with an LVEF of $40 \%$ (Fig. 2c), which was improved compared with that observed on the 11th hospital day. The patient was discharged from our hospital on the 23rd hospital day. Thereafter, she experienced no chest symptoms, and echocardiography performed six months after admission showed only slight impairment of the LV wall motion, with an LVEF of $51 \%$.

\section{Discussion}

In this study, we described the case of a patient with heart failure accompanied by chest pain and elevations in the levels of cardiac biomarkers. Because we believed that myocardial ischemia contributed to the patient's condition, we performed emergency coronary angiography; however, no sig- 
nificant coronary stenosis was observed on coronary angiography. Treatment for heart failure was initiated, although the patient's condition only gradually improved. Following stabilization of the heart failure, a second cardiac catheterization revealed the presence of multivessel coronary spasms on coronary angiography and ischemic changes within the endomyocardium, suggesting that the coronary spasms played some role in the development of heart failure in this patient.

There have been several reports investigating the relationship between heart failure and coronary spasms (5-12). The frequency of the provocation of coronary spasms in patients with LV dysfunction ranges from $2.4 \%$ to $47.6 \%(5-9,12)$. Although the frequency of coronary spasms in such patients may vary according to differences in the studied populations, the timing of the provocation of spasms and prescribed medications, recent reports suggest that the frequency of coronary spasms in patients with LV dysfunction is more common, ranging from $33 \%$ to $47.6 \%$ (5-8). In contrast, it has been reported that the coronary endothelial function is more severely impaired in patients with heart failure $(13,14)$, with coronary spasms being a consequence of heart failure. The finding that the occurrence of chest symptoms before the development of heart failure is less frequently observed (approximately 30\%) in patients with heart failure and coronary spasms $(7,8)$ may account for this phenomenon. However, several studies have demonstrated the effectiveness of antivasospastic agents, such as calciumchannel blockers, in improving the parameters of the cardiac function in patients with LV dysfunction and coronary spasms $(5,7,8,10,11)$. Sueda et al. (7) reported that the LV end-diastolic and end-systolic dimensions after one year of medical therapy are significantly smaller in patients with heart failure due to coronary spasms. Considering the effects of antivasospastic agents in improving the parameters of the cardiac function, coronary spasms may be a cause of heart failure.

In the present case, an endomyocardial biopsy revealed slight myocardial degeneration and interstitial fibrosis, indicating the possibility of essential myocardial disease. Inami et al. (8) reported no specific findings on endomyocardial biopsies in their study of patients with LV dysfunction and coronary spasms. In contrast, some experimental studies using animals have shown that microvascular spasms can cause myocardial degeneration and interstitial fibrosis (15). Therefore, coronary spasm-related changes in the myocardium may be present in the clinical setting, and the frequency of these changes may be variable due to the degree and duration of coronary spasm-induced myocardial ischemia. In the present case, we carefully focused more on the findings of the endomyocardium, which was obviously irregular and accompanied by slight lamellar fibrosis with differences in size, nuclear form irregularities and an arrangement disorder of the cardiac muscle cells under the endomyocardium. Although these findings are also nonspecific to cardiomyopathy, they are believed to indicate ischemia- related changes in the myocardium and endomyocardium due to chronic myocardial ischemia (16). This is because the myocardium on the endomyocardial side is more vulnerable to ischemia than that on the epicardial side, and disorders of the former appear earlier due to coronary circulatory disturbances. Considering these disorders of the endomyocardium and myocardium under the endomyocardium, extensive and chronic myocardial ischemia due to coronary spasms may, in part, contribute to the development of heart failure.

In this case, a calcium channel blocker was administered after a spasm provocation test, and the patient recovered without any chest symptoms or recurrence of heart failure. Regarding the patient's cardiac function, the LVEF on echocardiography was restored to $51 \%$ six months after discharge. Moreover, although the cardiac function may improve only with conventional treatment for heart failure, improvements in the cardiac function may be increased by combining conventional treatment for heart failure with the administration of antivasospastic agents, such as calcium channel blockers $(7,8)$. In addition, the use of monotherapy with a beta-blocker in patients with heart failure and positive spasms may sometimes result in the aggravation of the coronary spasms. Therefore, physicians should keep in mind the possibility of spasm-related ischemic heart failure, which was reported as vasospastic heart failure by Sueda et al. (7), and perform spasm provocation tests in patients with heart failure of unknown origin without fixed coronary stenosis.

In summary, we evaluated a woman with heart failure without significant stenosis on the first coronary angiogram in whom spasm-induced myocardial ischemia was diagnosed based on the findings of a second coronary angiogram and an endomyocardial biopsy. The coronary spasms may, in part, have contributed to the development of heart failure in this case. Coronary spasms should be considered a possible cause of heart failure.

\section{The authors state that they have no Conflict of Interest (COI).}

\section{References}

1. Yasue H, Kugiyama K. Coronary spasm: clinical features and pathogenesis. Intern Med 36: 760-765, 1997.

2. Yasue H, Nakagawa H, Itoh T, Harada E, Mizuno Y. Coronary artery spasm--clinical features, diagnosis, pathogenesis, and treatment. J Cardiol 51: 2-17, 2008.

3. Hung MJ. Current advances in the understanding of coronary vasospasm. World J Cardiol 2: 34-42, 2010.

4. Takagi Y, Yasuda S, Tsunoda R, et al. Clinical characteristics and long-term prognosis of vasospastic angina patients who survived out-of-hospital cardiac arrest: multicenter registry study of the Japanese Coronary Spasm Association. Circ Arrhythm Electrophysiol 4: 295-302, 2011.

5. Sakata K, Nawada R, Ohbayashi K, Tamekiyo H, Yoshida H. Diffuse and severe left ventricular dysfunction induced by epicardial coronary artery spasm. Angiology 51: 837-847, 2000.

6. Nishi I, Iida K, Kawano S, et al. Effects of anti-vasospastic agents in Japanese patients with dilated cardiomyopathy and coronary vasospasm. Jpn Heart J 43: 333-342, 2002.

7. Sueda S, Kohno H, Oshita A, Izoe Y, Nomoto T, Fukuda H. Vaso- 
spastic heart failure: multiple spasm may cause transient heart failure? J Cardiol 54: 452-459, 2009.

8. Inami T, Kataoka M, Shimura N, et al. Left ventricular dysfunction due to diffuse multiple vessel coronary artery spasm can be concealed in dilated cardiomyopathy. Eur J Heart Fail 14: 11301138,2012

9. Nosaka H, Nobuyoshi M. Coronary arterial spasm and symptomatology in ischemic and non-ischemic heart diseases: study of the ergonovine maleate provocative test in 3,000 consecutive patients. J Cardiogr Suppl 12: 35-47, 1987 (Abstract in English).

10. Figulla HR, Rechenberg JV, Wiegand V, Soballa R, Kreuzer H Beneficial effects of long-term diltiazem treatment in dilated cardiomyopathy. J Am Coll Cardiol 13: 653-658, 1989.

11. Packer M, O'Connor CM, Ghali JK, et al. Effect of amlodipine on morbidity and mortality in severe chronic heart failure. Prospective Randomized Amlodipine Survival Evaluation Study Group. N Engl J Med 335: 1107-1114, 1996.
12. Sueda S, Ochi N, Kawada H, et al. Frequency of provoked coronary vasospasm in patients undergoing coronary arteriography with spasm provocation test of acetylcholine. Am J Cardiol 83: 1186-1190, 1999.

13. Mathier MA, Rose GA, Fifer MA, et al. Coronary endothelial dysfunction in patients with acute-onset idiopathic dilated cardiomyopathy. J Am Coll Cardiol 32: 216-224, 1998.

14. Ninomiya Y, Hamasaki S, Ishida S, et al. Elevated levels of brain natriuretic peptide as a predictor of impaired coronary endothelial function in patients with left ventricular remodeling. J Cardiol 48: 125-132, 2006.

15. Factor SM, Sonnenblick EH. Hypothesis: is congestive cardiomyopathy caused by a hyperreactive myocardial microcirculation (microvascular spasm)? Am J Cardiol 50: 1149-1152, 1982.

16. Virmani R. Cardiomyopathy, ischemic. Bloom S, Ed. Lippincott Williams \& Wilkins, Philadelphia, 1996: 28-29.

(C) 2014 The Japanese Society of Internal Medicine http://www.naika.or.jp/imonline/index.html 